\title{
Longitudinal Vibrations of the Viscoelastic Moving Belt
}

\author{
Xiaoxu Pang, Junxia Li, and Ziming Kou \\ School of Mechanical Engineering, Taiyuan University of Technology, Shanxi Provincial Engineering Laboratory \& \\ Research Center for Mine Fluid Control, Taiyuan 030024, China \\ Correspondence should be addressed to Junxia Li; bstljx@163.com
}

Received 20 November 2014; Revised 23 December 2014; Accepted 6 January 2015

Academic Editor: Tony Murmu

Copyright (C) 2015 Xiaoxu Pang et al. This is an open access article distributed under the Creative Commons Attribution License, which permits unrestricted use, distribution, and reproduction in any medium, provided the original work is properly cited.

The longitudinal dynamic governing equation of the viscoelastic belt with one end subjected to concentrated mass was established based on the Kelvin-Voigt viscoelastic partial-differential constitutive law. The generalized coordinate method was adopted to solve dynamic displacement and dynamic tension. And then it was reduced to be a nonhomogeneous partial-differential equation where the analytical solutions with a constant acceleration were obtained. The effects of damping coefficient, the loading radio, and the constant acceleration of the belt on the dynamic response of the belt were investigated using the established dynamic model. The results show that the longitudinal vibration frequency of the viscoelastic moving belt increases with an increasing of the mass at the end. The increasing value of the loading radio, damping coefficient, and decreasing the acceleration will lead to a deceasing in dynamic tension. Moreover, the method of solution can be applied to axially moving viscoelastic materials with different boundary conditions.

\section{Introduction}

Viscoelastic belts have been widely used in power transmission system, especially in conveyors, and replace more expensive and complicated cars and trains. With the development of engineering materials, belts are usually composed of some metallic reinforcement materials such as steel-cords and polymeric materials such as rubbers, which makes the belts exhibit viscoelastic properties. To accurately describe the characters of the viscoelastic belt, its vibration analysis has been carried out to be studied extensively based on the viscoelastic constitutive law $[1,2]$. Wickert and Mote analyzed the nonlinear vibrations and the dynamic stability of the axially moving materials and obtained the response to arbitrary excitation and initial conditions in closed form $[3,4]$. Chen and Ding used the finite difference method to investigate the steady-state response of transverse forced vibration of an axially moving beam under the fixed boundary condition [5]. Ding and Chen used multiple scales to study the nonlinear partial-differential equation and the integropartial differential equation of transverse vibration of axially moving viscoelastic beams subjected to external transverse loads via steady-state periodical response $[6,7]$. Zhang and $\mathrm{Zu}$ investigated the nonlinear vibration and stability of the parametrically excited viscoelastic string by multiple scales. In their paper, the constitutive law of the Kelvin model was used and the governing equation was obtained $[8,9]$. Pakdemirli and Ozkaya obtained a boundary layer solution for an axially moving string using multiple scales [10]. Shin et al. discretized the governing equations and investigated the effects of system parameters on the natural frequencies, mode shapes, and stability [11]. Chen and Yang studied the time-variant velocity of the axially moving viscoelastic belt [12]. Chen et al. obtained the transverse vibration of the axially moving belt through the modified finite difference [13]. Chen employed the Kelvin model and Euler-Bernoulli beam model to establish the nonlinear governing equation of the viscoelastic belt. In their paper, the periodic and chaotic oscillations of the parametrically excited the moving belt [14].

From all the above-mentioned investigations, it can be concluded that the majority of the investigations were devoted to the transversal vibration of the axially moving viscoelastic materials. However, the length of the moving belt is much larger than its width usually. Therefore, longitudinal vibration of the axially moving viscoelastic materials is more important, especially during the start and stop of the moving belt, such as conveyors. However, fewer papers discussed the longitudinal vibration of the viscoelastic moving belt. Even 
Kuz'menko and Sitnyanskii presented a simple method of linearizing nonlinear viscoelastic relations of the longitudinal viscoelastic vibrations for a rod with one end mass [15]. The authors did not give any application of the axially viscoelastic moving material. The governing equations of the viscoelastic moving belt containing a term of the mixed partial derivative with respect to the time and the space coordinate are a viscoelastic continuum. So, the related researches also have theoretical significance.

As exact values of the continuity equation are usually unavailable, approximate analytical methods, multiple scales, and discrete element methods, that is, the finite element method [16-24], are widely used to investigate vibration of the belt when we solved a concrete issue. Then the abovementioned methods can be used to solve the governing equation. However, the accurate solution cannot be obtained from these methods.

In the paper, the dynamic governing equation of the viscoelastic moving belt with one end subjected to concentrated mass was established based on the Kelvin-Voigt viscoelastic partial-differential constitutive law. The generalized coordinate method was adopted to solve the accurate dynamic displacement and tension. The effects of system parameters, such as the loading radio, damping coefficient, and the acceleration, on the dynamic response of the viscoelastic moving belt were also investigated.

\section{Governing Equation}

A viscoelastic belt, with density of $\rho$, cross-sectional area of $A$, and mass of one end $M$, travels from speed 0 to $V$ at a constant axial acceleration $a(t)$ between one rigidly clamped end and the other end subjected to concentrated mass. The Kelvin-Voigt viscoelastic model was chosen to describe the viscoelastic properties of the viscoelastic belt in this paper. The model was composed of a linear spring and a linear dashpot. They were connected in parallel. The corresponding linear differential operator $\widehat{E}$ for Kelvin-Voigt viscoelastic model is

$$
\widehat{E}=E+\eta \frac{\partial}{\partial t}=E\left(1+\mu \frac{\partial}{\partial t}\right)
$$

where $E$ is the stiffness constant of the spring, $\eta$ is the dynamic viscosity of the dashpot, and $\mu=\eta / E$ is the retardation time.

The stress $\sigma(t)$ in the constitutive law is given as

$$
\sigma(t)=E \varepsilon+\eta \frac{\partial \varepsilon}{\partial t}=E\left(1+\mu \frac{\partial}{\partial t}\right) \varepsilon
$$

And the tension equation is obtained as

$$
S(x, t)=E A\left(1+\mu \frac{\partial}{\partial t}\right) \frac{\partial U}{\partial x},
$$

where $U$ represents the displacement of the cross-section, which includes the static displacement $w(x, t)$ and the dynamic displacement $u(x, t)$.

The transverse vibration is neglected. We can obtain the partial-differential equation of the longitudinal vibrations of the viscoelastic moving belt with an end subjected to concentrated mass as

$$
\rho d x\left(\frac{\partial^{2} U}{\partial t^{2}}+a(t)\right)=\frac{\partial S}{\partial x} d x+\rho g d x .
$$

Substituting (3) into (4) yields

$$
\frac{\partial^{2} U}{\partial t^{2}}-c^{2}\left(1+\mu \frac{\partial}{\partial t}\right) \frac{\partial^{2} U}{\partial x^{2}}=g-a(t)
$$

The boundary conditions and the initial conditions of (5) are as follows:

$$
\begin{gathered}
U(0, t)=0, \\
U(l, t)=w+u(l, t), \\
U(x, 0)=0, \\
\frac{\partial U(x, 0)}{\partial t}=0 .
\end{gathered}
$$

\section{Dynamic Response via the Generalized Coordinate Method}

3.1. Model Shape. As mentioned above (3), the displacement of the belt consists of the static displacement and the dynamic displacement. The static displacement is mainly caused by the weight of the belt and the concentrated mass; the static displacement could be written as

$$
w_{0}=\frac{M+\rho l g}{E A} x-\frac{\rho g}{2 E A} x^{2} .
$$

Substitution of (8) into (5) yields a dynamic partialdifferential equation

$$
\frac{\partial^{2} u}{\partial t^{2}}=c^{2}\left(1+\mu \frac{\partial}{\partial t}\right) \frac{\partial^{2} u}{\partial x^{2}}-a(t) .
$$

The boundary conditions of (9) are as follows:

$$
\begin{gathered}
u(0, t)=0 \\
M\left[\frac{\partial^{2} u(l, t)}{\partial t^{2}}+a(t)\right]=-E A\left(1+\mu \frac{\partial}{\partial t}\right) \frac{\partial u(l, t)}{\partial x} .
\end{gathered}
$$

The initial conditions would be the same with (7).

Equation (9) is nonhomogeneous differential equation of dynamic displacement; the Duhamel principle is employed to solve (9) via homogenizing:

$$
\frac{\partial^{2} u}{\partial t^{2}}=c^{2}\left(1+\mu \frac{\partial}{\partial t}\right) \frac{\partial^{2} u}{\partial x^{2}}
$$

For free vibration, the homogeneous boundary conditions of (11) are as follows:

$$
\begin{gathered}
M \frac{\partial^{2} u(l, t)}{\partial t^{2}}=-E A\left(1+\tau \frac{\partial}{\partial t}\right) \frac{\partial u(l, t)}{\partial x}, \\
u(0, t)=0 .
\end{gathered}
$$


TABLE 1: Value of $\eta$ corresponding to different $\alpha$.

\begin{tabular}{cccccccccc}
\hline$\alpha$ & 0.01 & 0.4 & 0.8 & 1.0 & 2.0 & 4.0 & 10 & 100 & 1000 \\
\hline$\eta_{1}$ & 0.10 & 0.60 & 0.79 & 0.86 & 1.08 & 1.27 & 1.43 & 1.56 & 1.57 \\
\hline$\eta_{2}$ & 3.15 & 3.26 & 3.37 & 3.43 & 3.64 & 3.94 & 4.31 & 4.67 & 4.71 \\
\hline$\eta_{3}$ & 6.29 & 6.35 & 6.41 & 6.44 & 6.58 & 6.81 & 7.23 & 7.78 & 7.85 \\
\hline$\eta_{4}$ & 9.43 & 9.47 & 9.51 & 9.53 & 9.63 & 9.81 & 10.20 & 10.89 & 10.99 \\
\hline
\end{tabular}

To investigate the dynamic response, the separation of variable is employed to solve (11):

$$
u(x, t)=X(x) T(t)
$$

where $X(x)$ is spatially function and $T(t)$ is temporally function.

Substitution (13) into (11) would lead to

$$
\frac{\ddot{T}}{c^{2}(T+\mu \dot{T})}=\frac{X^{\prime \prime}}{X}=-\lambda^{2} .
$$

From the boundary conditions, we can obtain the model shape

$$
\begin{aligned}
X_{m} & =b \sin \frac{\eta_{m}}{l} x, \\
\alpha & =\eta \tan \eta,
\end{aligned}
$$

where $\alpha=\rho l / M$ is the loading ratio of the belt's self-weight and the concentrated mass. $\eta_{m}=\lambda_{m} l$ is new parameter.

Equation (16) is a transcendental equation and it has infinite solutions. The values of $\eta$ which are corresponding to different values of $\alpha$ are shown in Table 1 .

A general solution of $T(t)$ is sought in the form of (14):

$$
T=e^{-\xi v_{m} t}\left(D_{1} \cos \omega_{m} t+D_{2} \sin \omega_{m} t\right),
$$

where $v_{m}=c \eta / l$ is the natural frequency, $\xi=\mu v_{m} / 2$ is damping coefficient, and $\omega_{m}=\alpha v_{m} \sqrt{1-\xi^{2}}$ is damped frequency.

3.2. Dynamic Displacement. In the following analysis, the generalized coordinate method is used to obtain the dynamic displacement of the viscoelastic belt. And the mathematical statement of the arbitrary cross-section dynamic displacement in nonsteady state is as follows

$$
u(x, t)=\sum_{m=1}^{\infty} X_{m}(x) q_{m}(t) .
$$

Based on the analysis above, the vibration mode $X(x)$ had been derived from the homogeneous equation as $X_{m}(x)=$ $\sin \left(\eta_{m} x / l\right)$. To solve the generalized coordinate of the time $q_{m}(t)$, the second Lagrange differential equation is given as

$$
\frac{d}{d t} \frac{\partial K}{\partial \dot{q}_{m}}-\frac{\partial K}{\partial q_{m}}+\frac{\partial R}{\partial \dot{q}_{m}}+\frac{\partial H}{\partial q}=Q_{m},
$$

where $K$ represents the kinetic energy, $R$ indicates the elastic strain energy or elastic potential energy, $H$ denotes the virtual work by both external and viscous dissipative force, $R=$ $-(1 / 2)(d / d t)(K+H)$, and $Q_{m}$ accounts for the generalized force.

The kinetic energy of the viscoelastic belt is given as

$$
K=\frac{\rho}{2} \int_{0}^{L} X^{2} \sum_{n=1}^{\infty} \dot{q}_{m} d x+\frac{M}{2}\left[\sum_{n=1}^{\infty} X^{2} \dot{q}_{m}^{2}\right] .
$$

The elastic strain energy is obtained as

$$
H=\frac{E A}{2} \sum_{n=1}^{\infty} q_{m}^{2} \int_{0}^{l} X^{\prime} d x .
$$

The dissipative energy is represented as

$$
R=\frac{\mu E A}{2} \sum_{n=1}^{\infty} \dot{q}_{m}^{2} \int_{0}^{l} X^{\prime} d x .
$$

Substituting (20), (21), and (22) into (19) yields

$$
\begin{gathered}
\ddot{q}_{m}+\frac{\tau c^{2} \int_{0}^{l} X^{\prime 2} d x}{\int_{0}^{l} X^{2} d x+(M / \rho) X^{2}(l)} \dot{q}_{m} \\
+\frac{c^{2} \int_{0}^{l} X^{\prime 2} d x}{\int_{0}^{l} X^{2} d x+(M / \rho) X^{2}(l)} q_{m}=\frac{Q_{m}}{\rho \int_{0}^{l} X^{2} d x+M X^{2}(l)} .
\end{gathered}
$$

From (23), (14), and (11) we can obtain

$$
\frac{\int_{0}^{l} X^{\prime 2}}{\int_{0}^{l} X^{\prime 2}+(M / \rho) X^{2}(l)}=\lambda^{2} .
$$

Substituting (24) into (23) yields

$$
\ddot{q}_{m}+\mu c^{2} \lambda^{2} \dot{q}+c^{2} \lambda^{2} q_{m}=-\frac{\lambda^{2} Q_{m}}{\rho \int_{0}^{l} X^{\prime 2} d x} .
$$

Equation (25) is applicable to various boundary conditions (both ends free; one end fixed, the other free, etc.); only the mode shapes $X(x)$ are different according to different boundary conditions.

The mathematical statement of the generalized force according to the principle of virtual work is given as

$$
Q_{m}=M a(t) X_{m}(l)+\int_{0}^{l} \rho a(t) X_{m} d x=\frac{\alpha M}{\eta_{m}} a(t) .
$$

The solution of (25) is expressed in the form

$$
q_{m}=\int_{0}^{t} \frac{v_{m}^{2}}{E A \int_{0}^{l} X^{\prime 2} d x} Q_{m}(\tau) \frac{e^{-\xi v_{m}(t-\tau)}}{\omega_{m}} \sin \omega_{m}(t-\tau) d \tau .
$$

Substituting (26) and (27) into (18) yields

$$
u(x, t)=\frac{\rho l^{2}}{E A} H_{m} \sum_{m=1}^{\infty} \frac{\sin \left(\eta_{m} x / l\right)}{\eta_{m}\left(1-\xi^{2}\right)} A(t),
$$


TABLE 2: The first four-order natural frequency.

\begin{tabular}{cccccccccc}
\hline$\alpha$ & 0.01 & 0.4 & 0.8 & 1.0 & 2.0 & 4.0 & 10 & 100 & 1000 \\
\hline$v_{1}$ & 0.46 & 2.76 & 3.63 & 3.96 & 4.97 & 5.84 & 6.58 & 7.18 & 7.22 \\
\hline$v_{2}$ & 14.49 & 15.00 & 15.50 & 15.78 & 16.74 & 18.12 & 19.82 & 21.48 & 21.67 \\
\hline$v_{3}$ & 28.93 & 29.21 & 29.49 & 29.62 & 30.27 & 31.33 & 33.26 & 35.79 & 36.11 \\
\hline$v_{4}$ & 43.39 & 43.56 & 43.75 & 43.84 & 44.30 & 45.13 & 46.92 & 50.09 & 50.55 \\
\hline
\end{tabular}

where

$$
\begin{gathered}
A(t)=\omega_{m} \int_{0}^{t} e^{-\xi \nu_{m}(t-\tau)} a(\tau) \sin \omega_{m}(t-\tau) d \tau, \\
H_{m}=\frac{4}{\eta_{m}\left(2 \eta_{m}+\sin 2 \eta_{m}\right)} .
\end{gathered}
$$

Substituting (28) into (3) yields

$$
s(x, t)=\rho A l \sum_{m=1}^{\infty} \frac{H_{m}}{1-\xi^{2}} \cos \frac{\eta_{m} x}{l}[A(t)+\mu \dot{A}(t)] .
$$

Equations (28) and (30) describe the longitudinal dynamic displacement and dynamic tension of the viscoelastic moving belt with the Kelvin-Voigt viscoelastic constitutive law, respectively.

\section{Analytical Results}

The length of the investigated belt was $10 \mathrm{~m}$ in this study. The cross-sectional area $A$, the stiffness constant $E$, and the density $\rho$ were $0.0002 \mathrm{~m}^{2}, 10^{11} \mathrm{~Pa}$, and $9.5 \times 10^{3} \mathrm{~kg} / \mathrm{m}^{3}$, respectively.

4.1. The Natural Frequency of the Moving Belt. The natural frequency of the moving belt was obtained based on (17) and Table 1 . The variations of the natural frequency value with loading ratio are shown in Table 2 . It can be seen that the natural frequency is not unique and has infinite discrete values; the natural frequency is the fundamental frequency for $m=1$. The fundamental frequency always dominates the free vibration and forced vibration of the system, and when the system starts, the fundamental frequency is the first resonant frequency, the first one to avoid or rush across, and thus the determination of fundamental frequency has an important role in the system analysis. In this study, the first four-order of the natural frequency was chosen to show the natural frequency under different loading ratio, which can indicate the vibration characteristics enough. From Table 2, we can summarize that the natural frequency of the moving belt increases with the loading ratio increasing.

When the end mass of the moving belt is much less than the belt's self-weight, namely, $\alpha \rightarrow \infty$, then $\tan \eta_{m} \rightarrow \infty$, and thus $v_{m}=(c / l)((2 n-1) / 2) \pi$ and the natural frequency of the belt reaches its highest value. Otherwise, when the end mass of the moving belt is much bigger than the belt's selfweight, namely, $\alpha \approx 0$, then $\tan \eta_{m}=\eta_{m}$, that was $v_{m}=$ $(c / l) \sqrt{\alpha}$, and the free vibration natural frequency of the belt is almost zero.

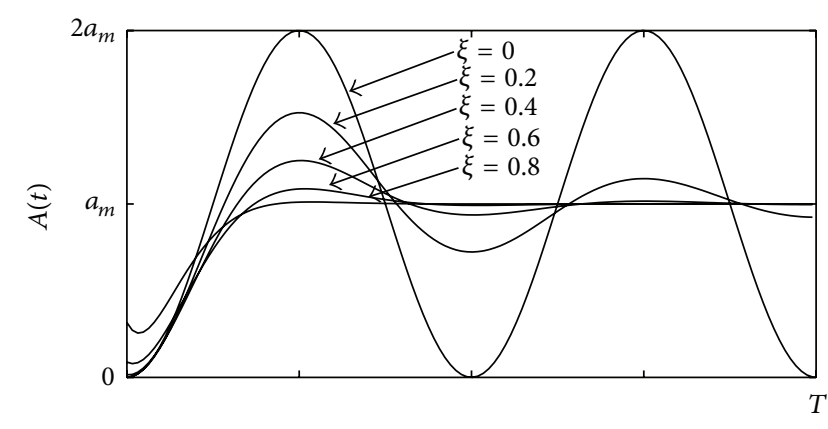

$t(\mathrm{~s})$

FIGURE 1: The influence of the damping coefficient on time response.

TABLE 3: The overshoot $M_{P} \%$ with different damping coefficient $\xi$.

\begin{tabular}{lcccccc}
\hline$\xi$ & 0 & 0.2 & 0.4 & 0.6 & 0.8 & 1 \\
\hline$M_{P} \%$ & 100 & 11.47 & 4.86 & 0.59 & 0.01 & 0 \\
\hline
\end{tabular}

4.2. Time Response. When the acceleration of the moving belt is constant, substituting $a(t)=a$ into (28) leads to the time response properties

$$
A(t)=a_{m}\left[1-\frac{e^{-\xi v_{m} t}}{\sqrt{1-\xi^{2}}} \cos \left(\omega_{m} t-\varphi\right)\right],
$$

where $\varphi=\tan ^{-1}\left(\xi / \sqrt{1-\xi^{2}}\right)$.

The effects of the constant acceleration on time response of the moving belt are given for $0 \leq \xi<1$ in Figure 1 . When $\xi$ was changed from zero to one, the effects of the constant acceleration of the moving belt on time response of the moving belt are shown in Figure 1.

Combining Figure 1 and (31), we can obtain the influence of the damping coefficient on time response with the condition of the constant acceleration. When the damping coefficient is zero, it is equivalent to the vibration of the elastic belt, namely, an undamped vibration with the continuous undergoing vibration. When the damping coefficient is in the span $0<\xi<1$, the vibration is subcritical damping vibration. Moreover, the maximum overshoot would decrease gradually with the damping coefficient increasing (Table 3 ). Meanwhile, the peak time would increase while adjusting time decreases. According to the performance index of the second order system, the maximum overshoot of the system is $M_{P}=\left(1 / \sqrt{1-\xi^{2}}\right) e^{-\left((\pi+4) \xi / \sqrt{1-\xi^{2}}\right)}$. With the increase of damping coefficient, the maximum overshoot would decrease and system stability would be improved. When $\xi$ is greater than 0.4 , the overshoot would be less than $5 \%$.

4.3. Dynamic Tension Response. In this section, the numerical simulation is presented to show the effectiveness of analytic solutions. The objectives of this section are to investigate the loading ratio, the damping coefficient, and the acceleration of the moving belt on the dynamic tension response curve at the rigidly clamped end. 


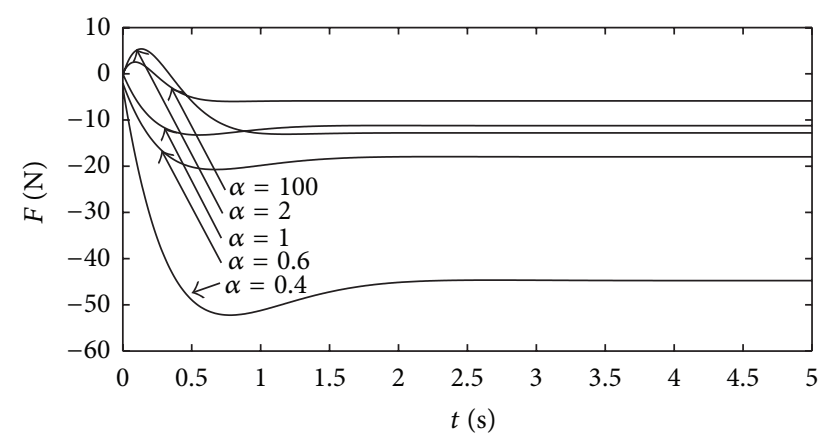

FIGURE 2: The influence of the loading ratio on the dynamic tension $(\xi=0.8, a=0.4 \mathrm{~m} / \mathrm{s})$ : (a) $\alpha=0.4$; (b) $\alpha=0.6$; (c) $\alpha=1$; (d) $\alpha=2$; (e) $\alpha=100$.

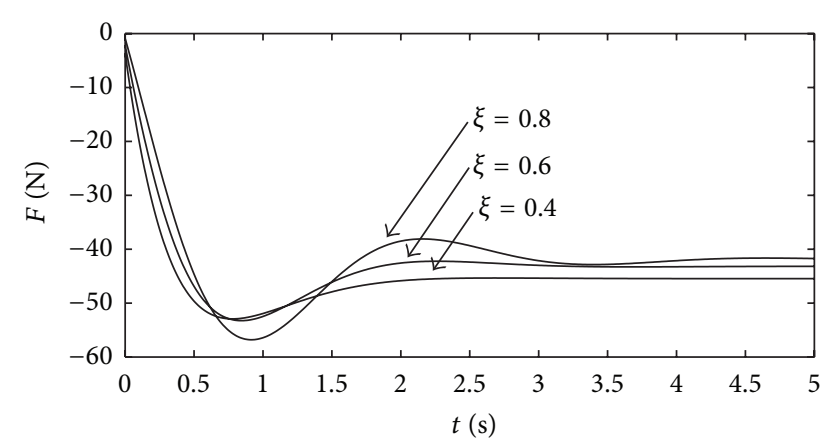

FIgURE 3: The influence of the damping coefficient on the dynamic tension ( $\alpha=0.4, a=0.4 \mathrm{~m} / \mathrm{s})$ : (a) $\xi=0.4$; (b) $\xi=0.6$; (c) $\xi=0.8$.

4.3.1. Effect of the Loading Ratio. Figure 2 shows the influence of the loading ratio on the longitudinal amplitudes of the moving belt, where the damping coefficient $\xi$ equals 0.8 and the acceleration $a$ is $0.4 \mathrm{~m} / \mathrm{s}$. It can be seen that the amplitude of dynamic tension and adjusting time become small when the loading ratio increases. The reason is that the elastic strain energy or elastic potential energy of the end mass will be smaller with increasing the loading radio, so the dynamic tension will decrease. The energy was indicated (23). When the value of loading ratio $\alpha$ is larger than one, there are positive and negative dynamic tension. The main reason is that when the value of loading ratio $\alpha$ is larger than one, the compressive stress appeared in the moving belt at the starting moment. With time increasing, the tensile stress appeared. It can be observed from Figure 2(d) $(\alpha=1)$ that the tension is zero at the starting moment; otherwise, the starting tensions of the left are not 0 .

4.3.2. Effect of the Damping Coefficient. The effects of the damping coefficient of the moving belt on dynamic tension are given in Figure 3, where the loading ratio $\alpha$ equals 0.4 and the acceleration $a$ is $0.4 \mathrm{~m} / \mathrm{s}$. The max values of the dynamic tension are $-55.9 \mathrm{~N},-51.1 \mathrm{~N}$, and $-49.2 \mathrm{~N}$. It can be seen that increasing the value of the damping coefficient $\xi$ leads to a deceasing in vibration amplitudes and adjusting time of the dynamic tension. The damping coefficient indicates the energy dissipation capability of the viscoelastic belt

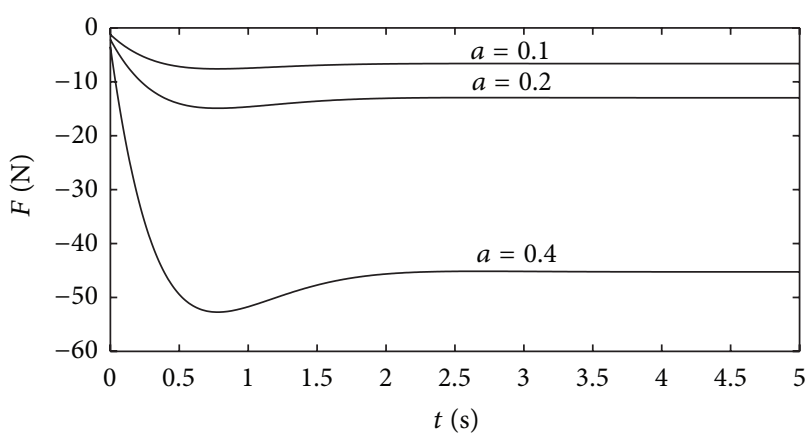

FIGURE 4: The influence of the acceleration on the dynamic tension $(\xi=0.8, \alpha=0.4)$ : (a) $a=0.1 \mathrm{~m} / \mathrm{s}$; (b) $a=0.2 \mathrm{~m} / \mathrm{s}$; (c) $a=0.4 \mathrm{~m} / \mathrm{s}$.

and furthest absorbs and consumes impact energy of the dynamic tension. The energy is just the dissipative energy as mentioned in (22). So the longitudinal amplitudes of the moving belt decrease with increasing the damping coefficient.

4.3.3. Effect of the Acceleration. The effects of the acceleration of the moving belt on the dynamic tension response curves are depicted in Figure 4 . The acceleration $a$ was chosen as $0.1 \mathrm{~m} / \mathrm{s}, 0.2 \mathrm{~m} / \mathrm{s}$, and $0.4 \mathrm{~m} / \mathrm{s}$, respectively. The $\max$ amplitudes of the dynamic tension are $-6.4 \mathrm{~N},-12.8 \mathrm{~N}$, and $-49.2 \mathrm{~N}$ in Figure 4 . We can observe that the stability of the dynamic response is improved and the dynamic tension amplitude decreases with the decreasing of the acceleration. There is one reason to clarify the result. The smaller the acceleration, the smaller the kinetic energy of the moving belt, so the longitudinal amplitudes of the dynamic tension will be smaller.

\section{Conclusions}

In this study, the accurate form of the governing equations of the viscoelastic belt with one end subjected to concentrated mass is derived using the generalized coordinate method. The effects of the system parameter of the moving belt on the dynamic response are investigated based on the derivative dynamic governing equation. The conclusions of this study are summarized as follows.

(1) The longitudinal vibration frequency of the viscoelastic belt will decrease with the increasing of the concentrated mass at one end.

(2) Increasing the value of the loading radio and damping coefficient and decreasing the acceleration of the moving belt will lead to a deceasing in dynamic tension at the rigidly clamped end.

(3) The method of solution can be applied to axially moving viscoelastic materials with different boundary conditions.

\section{Conflict of Interests}

The authors declare that there is no conflict of interests regarding the publication of this paper. 


\section{Acknowledgments}

The research is supported by the New Century Excellent Talents (Grant no. NECT-12-1038) and the Shanxi College of outstanding young academic leaders project (Grant no. 20120428). The first author gratefully acknowledges the helpful discussions with the research group and colleagues in the School of Mechanical Engineering at Taiyuan University of Technology.

\section{References}

[1] L. H. Chen, W. Zhang, and Y. Q. Liu, "Modeling of nonlinear oscillations for viscoelastic moving belt using generalized Hamilton's principle," Journal of Vibration and Acoustics, vol. 129, no. 1, pp. 128-132, 2007.

[2] L. Zhang and J. W. Zu, "Non-linear vibrations of viscoelastic moving belts, part I: free vibration analysis," Journal of Sound and Vibration, vol. 216, no. 1, pp. 75-90, 1998.

[3] J. A. Wickert and C. D. Mote Jr., "Classical vibration analysis of axially moving continua," Journal of Applied Mechanics, Transactions ASME, vol. 57, no. 3, pp. 738-744, 1990.

[4] J. A. Wickert and C. D. Mote Jr., "Current research on the vibration and stability of axially-moving materials," The Shock and Vibration Digest, vol. 20, no. 1, pp. 3-13, 1988.

[5] L. Q. Chen and H. Ding, "Steady-state transverse response in coupled planar vibration of axially moving viscoelastic beams," Transactions of the ASME-Journal of Vibration and Acoustics, vol. 132, no. 1, Article ID 0110091, 9 pages, 2010.

[6] H. Ding and L.-Q. Chen, "Approximate and numerical analysis of nonlinear forced vibration of axially moving viscoelastic beams," Acta Mechanica Sinica, vol. 27, no. 3, pp. 426-437, 2011.

[7] H. Ding and L.-Q. Chen, "Nonlinear models for transverse forced vibration of axially moving viscoelastic beams," Shock and Vibration, vol. 18, no. 1-2, pp. 281-287, 2011.

[8] L. Zhang and J. W. Zu, "Non-linear vibrations of viscoelastic moving belts, part II: forced vibration analysis," Journal of Sound and Vibration, vol. 216, no. 1, pp. 93-105, 1998.

[9] L. Zhang and J. W. Zu, "Non-linear vibrations of viscoelastic moving belts, part II: forced vibration analysis," Journal of Sound and Vibration, vol. 216, no. 3, pp. 93-105, 1998.

[10] M. Pakdemirli and E. Ozkaya, "Approximate boundary layer solution of a moving beam problem," Mathematical and Computational Applications, vol. 3, no. 2, pp. 93-100, 1998.

[11] C. Shin, J. Chung, and W. Kim, "Dynamic characteristics of the out-of-plane vibration for an axially moving membrane," Journal of Sound and Vibration, vol. 286, no. 4-5, pp. 1019-1031, 2005.

[12] L.-Q. Chen and X.-D. Yang, "Steady-state response of axially moving viscoelastic beams with pulsating speed: comparison of two nonlinear models," International Journal of Solids and Structures, vol. 42, no. 1, pp. 37-50, 2005.

[13] L.-Q. Chen, W.-J. Zhao, and J. W. Zu, "Simulations of transverse vibrations of an axially moving string: a modified difference approach," Applied Mathematics and Computation, vol. 166, no. 3, pp. 596-607, 2005.

[14] L. Q. Chen, "Principal parametric resonance of axially accelerating viscoelastic strings with an integral constitutive law," Proceedings of The Royal Society, vol. 461, no. 2061, pp. 42-66, 2005.
[15] V. A. Kuz'menko and N. A. Sitnyanskii, "Longitudinal viscoelastic vibrations of a rod with an end mass," Academy of Sciences of the Ukrainian SSR, vol. 5, no. 1, pp. 67-72, 1969.

[16] H. R. Öz, M. Pakdemirli, and H. Boyaci, "Non-linear vibrations and stability of an axially moving beam with time-dependent velocity," International Journal of Non-Linear Mechanics, vol. 36, no. 1, pp. 107-115, 2001.

[17] W. Zhang and C. Z. Song, "Higher-dimensional periodic and chaotic oscillations for viscoelastic moving belt with multiple internal resonances," International Journal of Bifurcation and Chaos, vol. 17, no. 5, pp. 1637-1660, 2007.

[18] M. H. Ghayesh and S. Balar, "Non-linear parametric vibration and stability of axially moving visco-elastic Rayleigh beams," International Journal of Solids and Structures, vol. 45, no. 25-26, pp. 6451-6467, 2008.

[19] M. H. Ghayesh, "Stability characteristics of an axially accelerating string supported by an elastic foundation," Mechanism and Machine Theory, vol. 44, no. 10, pp. 1964-1979, 2009.

[20] M. H. Ghayesh and S. Balar, "Non-linear parametric vibration and stability analysis for two dynamic models of axially moving Timoshenko beams," Applied Mathematical Modelling, vol. 34, no. 10, pp. 2850-2859, 2010.

[21] M. H. Ghayesh, "Parametric vibrations and stability of an axially accelerating string guided by a non-linear elastic foundation," International Journal of Non-Linear Mechanics, vol. 45, no. 4, pp. 382-394, 2010.

[22] L.-Q. Chen, Y.-Q. Tang, and C. W. Lim, "Dynamic stability in parametric resonance of axially accelerating viscoelastic Timoshenko beams," Journal of Sound and Vibration, vol. 329, no. 5, pp. 547-565, 2010.

[23] B. B. Özhan and M. Pakdemirli, "A general solution procedure for the forced vibrations of a system with cubic nonlinearities: three-to-one internal resonances with external excitation," Journal of Sound and Vibration, vol. 329, no. 13, pp. 2603-2615, 2010.

[24] K. Y. Sze, S. H. Chen, and J. L. Huang, "The incremental harmonic balance method for nonlinear vibration of axially moving beams," Journal of Sound and Vibration, vol. 281, no. 35, pp. 611-626, 2005. 

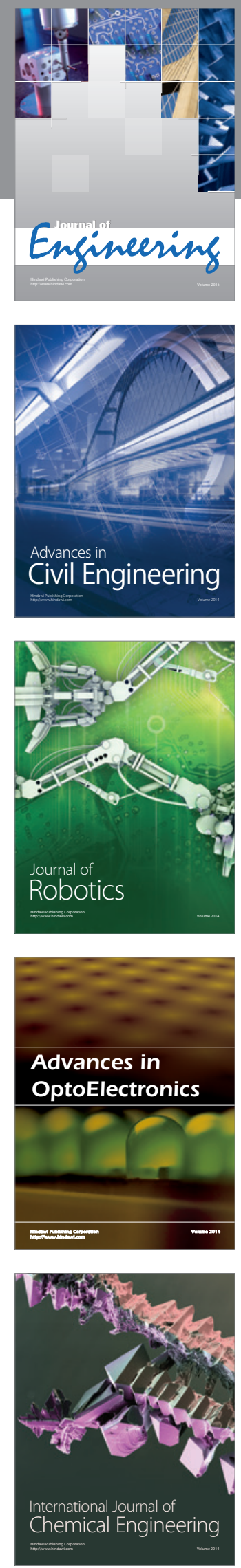

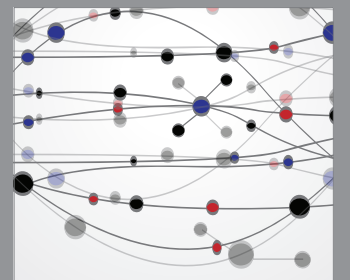

The Scientific World Journal
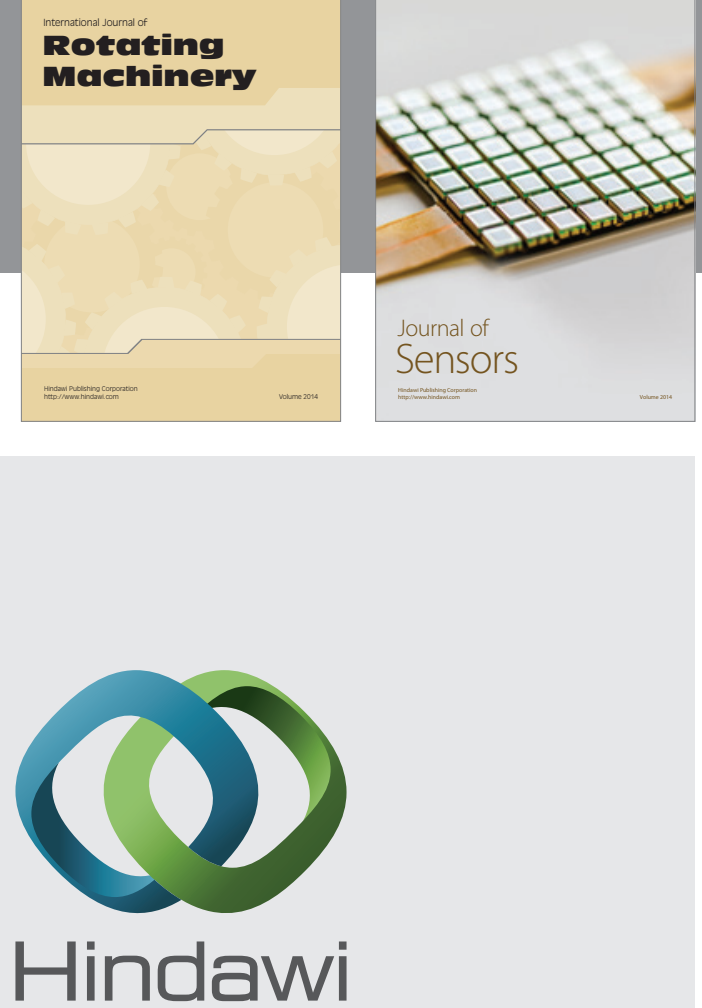

Submit your manuscripts at http://www.hindawi.com
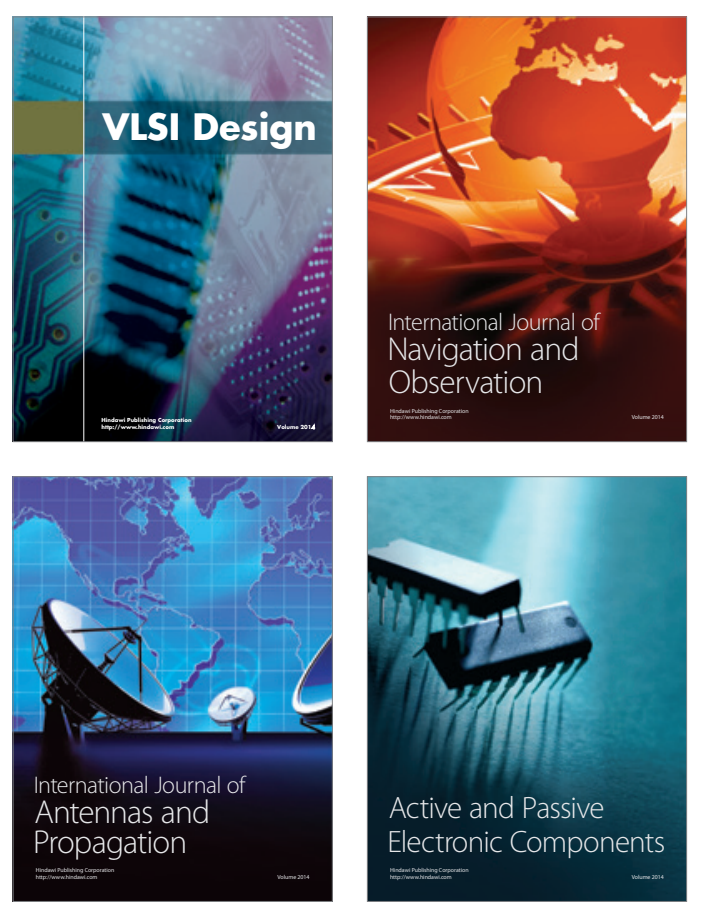
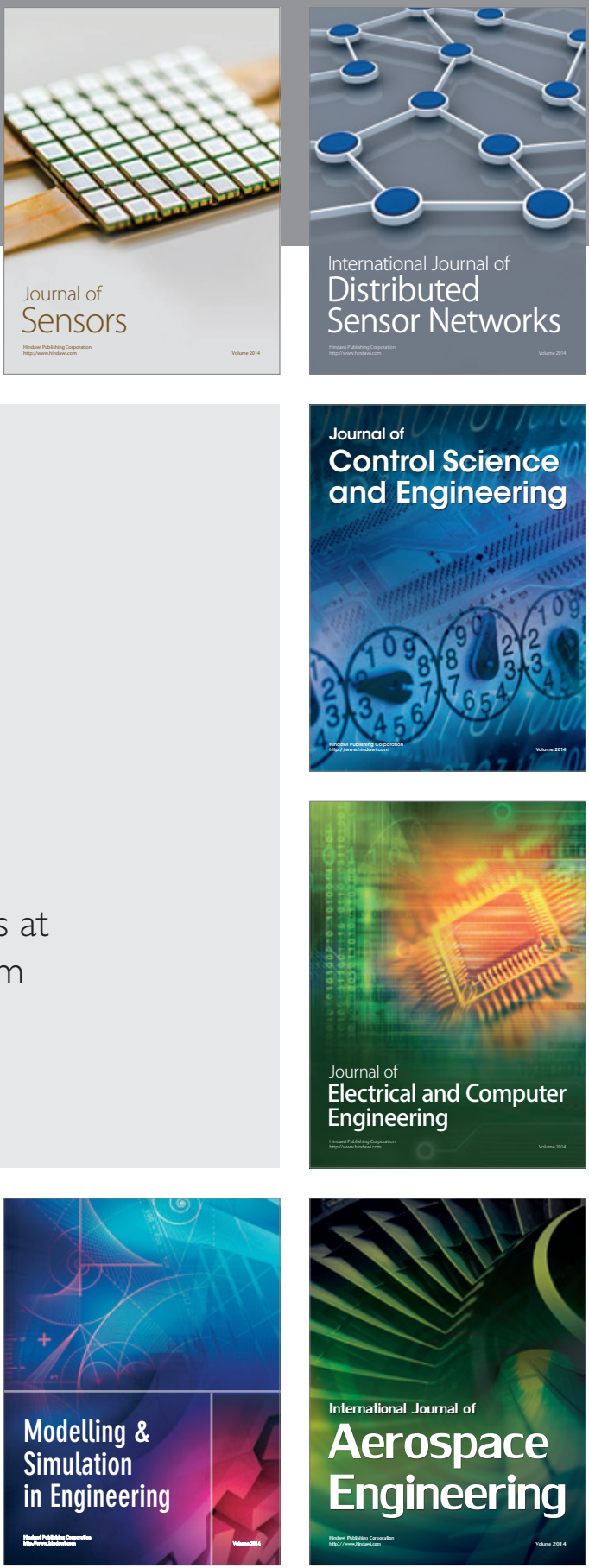

Journal of

Control Science

and Engineering
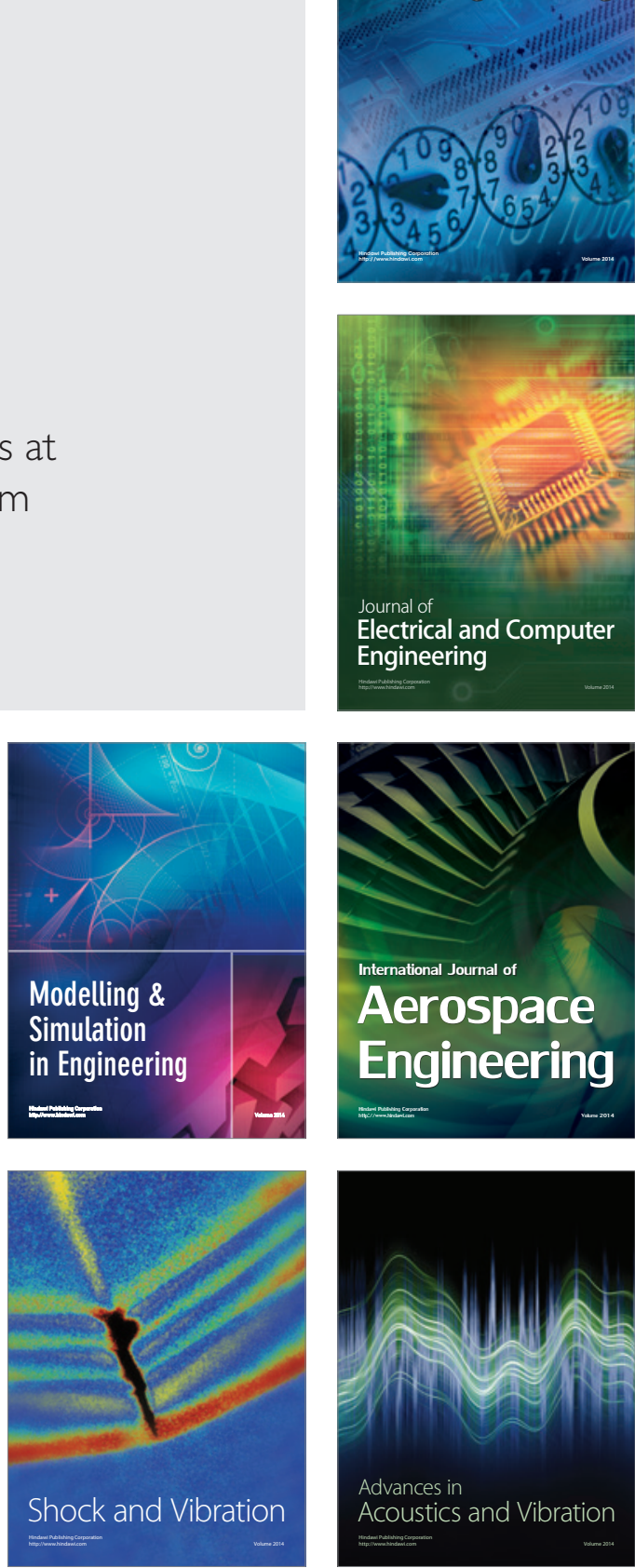